\title{
Benign Lymphadenopathy Presented in a Malignant Pathway - A Rare Case of Proteinaceous Lymphadenopathy
}

\author{
Md Serajul Islam ${ }^{1,2^{*}}$ and Pavel Kotoucek ${ }^{2}$ \\ ${ }^{1}$ Department of Haematology, Guys' \& St Thomas' Hospital, London, UK \\ ${ }^{2}$ Department of Haematology, Broomfield Hospital, Chelmsford, UK
}

*Corresponding author: Md Serajul Islam, Department of Haematology, Guys' \& St Thomas' Hospital, London; Department of Haematology, Broomfield Hospital, Chelmsford, UK, Tel: +44-7769580452, Fax: +44-1245-51-6669, E-mail: serajul@doctors.org.uk

\begin{abstract}
Proteinaceous lymphadenopathy is a rare non-specific lymph node changes incidence of which increases with age. This condition is usually associated with hypergammaglobulinemia or rheumatological conditions. However, this condition can also be due to unknown aetiology as we are presenting a case where no underlying case was found. It may affect nodal function, and rarely may it calcify. Histopathologist needs to be vigilant of this condition as lymphoma with lymph node sclerosis should be considered as differential diagnosis when patient present with this condition.
\end{abstract}

\section{Keywords}

Proteinaceous lymphadenopathy, Hypergammaglobulinemia, Rheumatological, Lymphoma

\section{Introduction}

Lymphadenopathy is a common clinical presentation in haematology outpatient departments. It is a cause of concern for the patient and physician alike even in the absence of symptoms. Proteinaceous lymphadenopathy also known as Hyaline deposition of lymph node is a rare condition and it is often a nonspecific finding. Its incidence increases with age $[1,2]$ and may reduce nodal function. A study has shown two distinct types of lymph node hyalinization-mediastinal-type hyalinisation where onion-peel lesions and associated meshwork areas were often found in the medullary sinus of the thoracic node and pelvic-type hyalinisation-where eosinophilic, glassy and spotty lesions were consistently seen in B lymphocyte areas of the pelvic node [2]. This difference seemed to be consistent with a difference in composite collagen fibrils of vascular walls between the thoracic and pelvic regions. With aging the incidence of hyalinisation increases and it seems that large proportion in total volume of nodes being involved [2]. The hyalinization seems to be one of the major events that diminish the nodal filtration function and ruin the node with aging [2]. Literature shows that this histological lymph node finding is usually associated with other conditions like rheumatological conditions and hypergammaglobulinemia $[3,4]$. Here we report a case of proteinaceous lymphadenopathy where no obvious aetiology was found and to our knowledge this is the first such case where this condition is reported without any underling associated condition.

\section{Case Report}

A 52-year-old man was seen in our department as an urgent suspected cancer referral to exclude lymphoma. Patient presented with 4 months history of swelling of left groin and left upper leg with any other specific symptoms; particularly he denied any weight loss, no fever, no history of infection and skeletal pain. He stated that the swelling was gradually getting bigger and it was causing some discomfort in his leg. Apart from treated hypertension he had no other past medical history. His physical examination confirms a mass of $20 \times 12 \mathrm{~cm}$ in diameter occupying his left upper thigh with no other palpable mass. Physical examination was otherwise unremarkable. His blood result showed Haemoglobin $125 \mathrm{~g} / \mathrm{l}$, white blood count 5.9 $\times 10^{9} / \mathrm{L}$ with normal differential white cell count, platelet count $341 \times 10^{9} / \mathrm{L}$, normal liver profile as well as renal profile. His adjusted calcium was normal at $2.36 \mathrm{mmol} / \mathrm{L}$, nor-

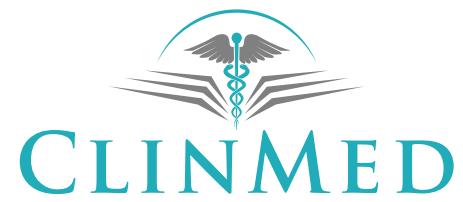

INTERNATIONAL LIBRARY

Citation: Islam MS, Kotoucek P (2018) Benign Lymphadenopathy Presented in a Malignant Pathway - A Rare Case of Proteinaceous Lymphadenopathy. Int J Blood Res Disord 5:030. doi.org/10.23937/24695696/1410030

Accepted: June 28, 2018: Published: June 30, 2018

Copyright: (c) 2018 Islam MS, et al. This is an open-access article distributed under the terms of the Creative Commons Attribution License, which permits unrestricted use, distribution, and reproduction in any medium, provided the original author and source are credited. 


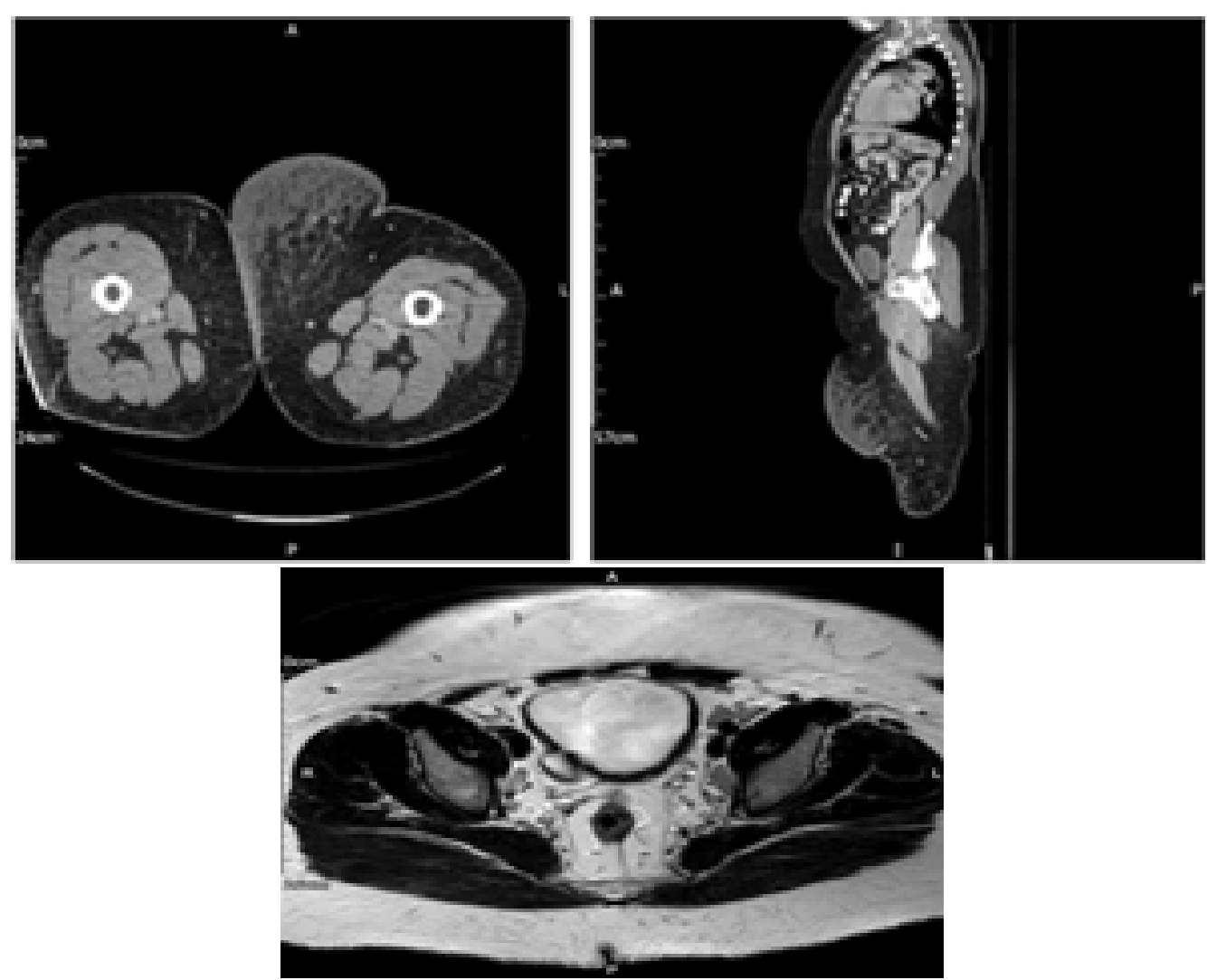

Figure 1: CT scan showed large swelling due to subcutaneous oedema (above) and MRI pelvis showing urinary Bladder diverticula (below).

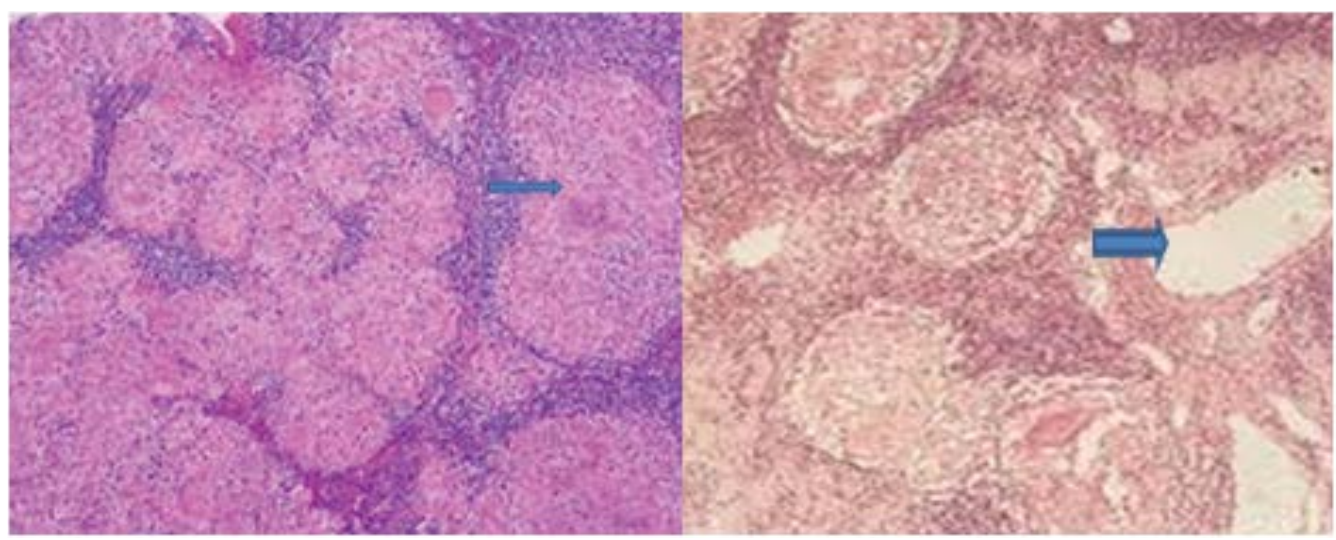

Figure 2: Thin arrow-H\&E stain, thick arrow-negative Congo Red stain. H\&E staining showed hyaline deposition of lymph node without any clonal plasma cell (thin arrow) \& Negative Congo red stain for amyloid (thick arrow).

mal Lactate Dehydrogenase (LDH) $264 \mathrm{iu} / \mathrm{L}$ and C-reactive protein normal. He had a CT scam of the abdomen and pelvis which showed normal intra-abdominal organs, one cystic mass in right inguinal fossa of $5.3 \times 3 \mathrm{~cm}$ in diameter. There was also bi-lateral inguinal lymphadenopathy with maximum size of $4.5 \mathrm{~cm}$. He also had a cystic swelling of $20 \times 12 \mathrm{~cm}$ diameter in his left upper thigh/left groin. Subsequently he had a magnetic resonance imaging (MRI) scan of pelvis which confirmed the above finding and also showed urinary bladder diverticula and computerised tomography scan (CT scan) showed large cystic swelling of left thigh was due to subcutaneous oedema (Figure 1). He did not undergo any investigation for his subcutaneous oedema of left upper thigh. However, fortunately his oedema improved with oral furosemide on subsequent out-patient visits.
Multiple large core biopsies were obtained from the largest left inguinal lymph node which showed deposition of eosinophilic hyaline material and the possibility of this eosinophilic hyaline material being amyloid has been excluded by negative Congo red stain (Figure 2). These samples also did not show any metastatic carcinoma or any lymphoma on haematoxylin \& eosin (H\&E) staining. It also did not show any vascular involvement of lymph nodes or any hyalinisation of vessels of lymph node. Rheumatological blood tests anti-CCP antibodies $1.1 \mathrm{U} / \mathrm{ml}$ was within normal range and connective tissue disease (CTD) Screen was also normal at 0.5 . His serum immunoglobulin levels were normal; no monoclonal band was detected on electrophoresis. Serum light chain analysis showed slightly raised kappa free light chain $32.84 \mathrm{mg} / \mathrm{L}$ (range 3.3-19.4 $\mathrm{mg} / \mathrm{L}$ ), normal lambda free light chain $7.65 \mathrm{mg} / \mathrm{L}$ (range 
5.7-26.3 mg/L). Virology screens were all negative. His liver function tests were all normal. Complement levels were not evaluated. Hence no obvious cause for the histological finding of hyaline deposition of lymph node was identified in our patient. Urinary bladder diverticula were thought be an incidental finding in our patient as there was no back flow or any significant pressure was detected on CT scan or MRI scan in this patient.

\section{Discussion}

Proteinaceous lymphadenopathy usually associated with rheumatoid arthritis and systemic sclerosis [3,4]. It appears that there is a good clinical response in RA-associated proteinaceous lymphadenopathy following successful treatment of arthritis $[3,4]$. It has also been reported in infective condition like HIV or Kikuchi's disease [5]. It has also been seen in association with hypergammaglobulinemia $[6,7]$ and post-treatment changes for carcinoma [8]. The histopathologist should be familiar with the morphological appearances of this condition, which can be confused with amyloidosis.

Proteinaceous lymphadenopathy of unknown aetiology is an exceedingly rare condition and we could not find any case without underlying cause or association in the literature. Described primarily as a pathologic entity, relatively little is known about its clinical manifestations or its response to therapy. The disease is often referred to and treated as an unusual form of plasma cell dyscrasia or light chain deposition disease [9]. Contrary to the notion that this condition is a clonal disorder [9] we found no obvious evidence of clonality in this patient. A Case of Proteinaceous lymphadenopathy was reported in literature [9] where the authors named that as angiocentric sclerosing lymphadenopathy (ASL) with proteinaceous lymphadenopathy with hyper-gammaglobulinemia (PLWH)-a polyclonal Systemic, non-amyloid deposition disorder. However, our patient did show normal immunoglobulin levels and there was no vascular hyaline deposition in vessels in lymph node biopsy. Contrary to that reported case our patient has normal liver function test although we did not check complement level in our patient which was found to be low in that reported case [9]. Slightly raised Kappa light chain may suggest autoimmune process and patient has been referred to rheumatology team.

Malignant lymphoma with sclerosis is the most important consideration in the differential diagnosis. Extensive lymph node hyalinisation is particularly common in the inguinal and iliac group of nodes as was seen in our patient [3]. Hyalinisation of lymph node can also be seen following inflammatory conditions such as sarcoidosis [10] or tuberculosis which should in mind when evaluating such conditions [11]. These changes may result from longstanding non-specific low-grade inflammation [12]. Occasionally extensive lymph node hyalinisation may be found with no clue as to its possible aetiology as seen in our case hence we suggest that there is entity of proteinaceous lymphadenopathy of unknown aetiology. This hyaline material may calcify, in time.

\section{Conclusion}

Proteinaceous lymphadenopathy which is usually a benign condition that occurs in association rheumatological conditions, infections and hypergammaglobulinemia. Incidence increases with age [1,2]. Malignant lymphoma with lymph node sclerosis and amyloid deposition should be considered in differential diagnosis.

\section{Funding}

Nothing to declare.

\section{Author Contribution}

MSI has conceived the articles, reviewed literature and written the initial draft of the manuscript, PK edited the manuscript, reviewed literature. Both author agreed on the final content of the manuscript.

\section{References}

1. Tsakraklides V, Tsakraklides E, Good RA (1975) An autopsy study of human axillary lymph node histology. Am J Pathol 78: 7-22.

2. Taniguchi I, Murakami G, Sato A, Fujiwara D, Ichikawa H, et al. (2003) Lymph node hyalinization in elderly Japanese. Histol Histopathol 18: 1169-1180.

3. McCluggage WG, Bharucha H (1994) Lymph node hyalinisation in rheumatoid arthritis and systemic sclerosis. J Clin Pathol 47: 138-142.

4. Al Rikabi AC, Naddaf HO, Al Balla SR, Al Sohaibani MO (1995) Proteinaceous lymphadenopathy in a patient with known rheumatoid arthritis--case report and review of the literature. Br J Rheumatol 34: 1087-1089.

5. Cossu A, Lissia A, Cossu S, Marras V, Tanda F, et al. (1998) Kikuchi's disease and proteinaceous lymphoadenopathy in 4 patients with HIV infection. Pathologica 90: 20-26.

6. Banerjee D, Mills DM, Hearn SA, Meek M, Turner KL (1990) Proteinaceous lymphadenopathy due to monoclonal nonamyloid immunoglobulin deposit disease. Arch Pathol Lab Med 114: 34-39.

7. Osborne BM, Butler JJ, Mackay B (1979) Proteinaceous lymphadenopathy with hypergammaglobulinemia. Am J Surg Pathol 3: 137-145.

8. Aktepe F, Kapucuoğlu N, Pak I (1996) The effects of chemotherapy on breast cancer tissue in locally advanced breast cancer. Histopathology 29: 63-67.

9. Michaeli J, Niesvizky R, Siegel D, Ladanyi M, Lieberman $\mathrm{PH}$, et al. (1995) Proteinaceous (Angiocentric sclerosing) lymphadenopathy: A polyclonal systemic, non-amyloid deposition disorder. Blood 86: 1159-1162.

10. Gümüştaş S, Inan N, Akansel G, Başyïğït I, Cïftçi E (2013) Differentiation of lymphoma versus sarcoidosis in the setting of mediastinal-hilar lymphadenopathy: Assessment with diffusion-weighted MR imaging. Sarcoidosis Vasc Diffuse Lung Dis 30: 52-59.

11. Subramanian $S$, Sandeepa HS, Chaudhari $P$, Kate AH, Kumar S, et al. (2014) Mediastinal lymphadenopathy in malignancy: Metastatic or granulomatous? J Assoc Physicians India 62: 630-632.

12. Li SL, Han JD (2017) Solitary nodule of angiolymphoid hyperplasia with eosinophilia of the back masquerading as pyogenic granuloma. Mol Clin Oncol 7: 874-876. 